QUARTERLY OF APPLIED MATHEMATICS

VOLUME LXIII, NUMBER 2

JUNE 2005, PAGES 335-341

S $0033-569 X(05) 00960-5$

Article electronically published on April 11, 2005

\title{
ON THE VISCOUS CAUCHY PROBLEM AND THE EXISTENCE OF SHOCK PROFILES FOR A $p$-SYSTEM WITH A DISCONTINUOUS STRESS FUNCTION
}

\author{
BY \\ JOÃO-PAUlO DIAS (CMAF/UL, Av. Prof. Gama Pinto, 2, 1649-003 Lisboa - Portugal) \\ AND \\ MÁRIO Figueira (CMAF/UL, Av. Prof. Gama Pinto, 2, 1649-003 Lisboa - Portugal)
}

Abstract. In this paper, we study the existence of weak solutions for the Cauchy problem and the existence of shock profiles for the system in viscoelasticity,

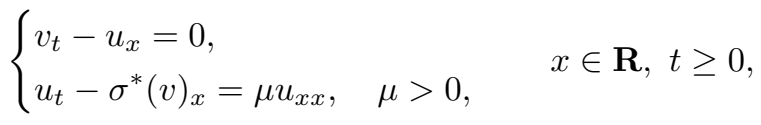

with $\sigma^{*}(v)=\sigma(v)+H(v)$, where $\sigma$ is a smooth stress function and $H$ is the usual Heaviside function. These kinds of models are motivated by some problems in mechanics of solids. Finally we solve, in related situations, the Riemann problem for the corresponding hyperbolic system.

1. Introduction and main results. The existence (and stability) of shock profiles for the system in viscoelasticity

$$
\left\{\begin{array}{l}
v_{t}-u_{x}=0, \\
u_{t}-\sigma(v)_{x}=\mu u_{x x}, \quad \mu>0, \quad x \in \mathbf{R}, t \geq 0,
\end{array}\right.
$$

has been studied in [10] for nonconvex smooth stress functions $\sigma$ such that

$$
\sigma(0)=0, \quad \sigma^{\prime}(0) \geq c>0, \quad \sigma^{\prime \prime}(v) v>0, v \in \mathbf{R} \backslash\{0\}
$$

$\left(\mathrm{ex}: \sigma(v)=v+\frac{v^{3}}{3}\right)$.

In some problems in mechanics of solids, such as the Savart-Masson effect (cf. [12], $\S 3.3 .2$, and [1], §4.31), it is reasonable to consider models where $\sigma$ is replaced by

$$
\sigma^{*}(v)=\sigma(v)+H(v), \quad v \in \mathbf{R}
$$

Received October 5, 2004.

2000 Mathematics Subject Classification. Primary 35L65.

E-mail address: dias@ptmat.fc.ul.pt

E-mail address: figueira@ptmat.fc.ul.pt

(C)2005 Brown University 
where $H(v)=1$ for $v>0$ and $H(v)=0$ for $v<0$ is the usual Heaviside function considered as the multi-valued function

$$
\widetilde{H}(v)=H(v) \quad \text { for } \quad v \neq 0, \quad \widetilde{H}(0)=[0,1] .
$$

A reasonable approximation of $H$ is given by $\int_{-\varepsilon}^{v} \rho_{\varepsilon}(y) d y$, with, for each $\varepsilon>0, \rho_{\varepsilon}(y)=$ $\frac{1}{\varepsilon} \rho\left(\frac{y}{\varepsilon}\right)$, where $\rho \geq 0$ is a $\mathcal{D}(\mathbf{R})=C_{c}^{\infty}(\mathbf{R})$ function with support $[-1,1]$ such that $\rho(-y)=$ $\rho(y)$ and $\int_{\mathbf{R}} \rho_{\varepsilon}(y) d y=1$ (cf. [5]). The functions $\rho_{\varepsilon}$ are the usual Friedrichs mollifiers and we have $\rho_{\varepsilon} \underset{\varepsilon \rightarrow 0}{\longrightarrow} \delta$ (Dirac distribution) in $\mathcal{D}^{\prime}(\mathbf{R})$. We set

$$
\sigma_{\varepsilon}(v)=\sigma(v)+\int_{-\varepsilon}^{v} \rho_{\varepsilon}(y) d y
$$

and we consider the approximate system

$$
\left\{\begin{array}{l}
v_{\varepsilon t}-u_{\varepsilon x}=0, \\
u_{\varepsilon t}-\sigma_{\varepsilon}\left(v_{\varepsilon}\right)_{x}=\mu u_{\varepsilon x x}, \quad x \in \mathbf{R}, t \geq 0 .
\end{array}\right.
$$

The Cauchy problem for systems of the type (1.5) has been studied in [8], 14] and [2] with the hypothesis

$$
\frac{\sigma(v)}{\Sigma(v)} \underset{|v| \rightarrow \infty}{\longrightarrow} 0, \quad \text { where } \quad \Sigma(v)=\int_{0}^{v} \sigma(y) d y
$$

and since

$$
\Sigma_{\varepsilon}(v)=\int_{0}^{v} \sigma_{\varepsilon}(y) d y \geq \Sigma(v), \quad v \in \mathbf{R},
$$

we get the following result (cf. [14] and [2]):

Proposition 1. Assume that the initial data $\left(v_{0}, u_{0}\right) \in\left(H^{2}(\mathbf{R})\right)^{2}$ and $v_{0} \in L^{1}(\mathbf{R})$. Then the Cauchy problem for the system (1.5) has a unique global solution

$$
\left(v_{\varepsilon}, u_{\varepsilon}\right) \in\left(C ^ { 1 } \left(\left[0,+\infty\left[; H^{2}\right) \cap C\left(\left[0,+\infty\left[; H^{2}\right)\right) \times\left(C ^ { 1 } \left(\left[0,+\infty\left[; L^{2}\right) \cap C\left(\left[0,+\infty\left[; H^{2}\right)\right)\right.\right.\right.\right.\right.\right.\right.\right.
$$

such that

$$
\begin{aligned}
\int_{\mathbf{R}}\left(v_{\varepsilon}^{2}+u_{\varepsilon}^{2}\right)(x, t) d x \leq c_{1}, \quad t \geq 0, \\
\mu \int_{0}^{t} \int_{\mathbf{R}}\left(v_{\varepsilon x}^{2}+u_{\varepsilon x}^{2}\right)(x, \tau) d x d \tau \leq c_{2}, \quad t \geq 0, \quad 0<\mu \leq 1, \\
\mu^{2} \int_{\mathbf{R}} v_{\varepsilon x}^{2}(x, t) d x \leq c_{3}, \quad t \geq 0
\end{aligned}
$$

with $c_{i}, i=1,2,3$, not depending on $\varepsilon$ (or on $\mu$ ).

REMARK. From (1.7) and (1.9) we get, for each $\mu$, a uniform (in $t$ and in $\varepsilon$ ) estimate for $\left\|v_{\varepsilon}(\cdot, t)\right\|_{L^{\infty}(\mathbf{R})}$.

Now, by the usual techniques in functional analysis, the well-known compact imbeddings of Sobolev spaces in bounded domains of $\mathbf{R}^{2}$ and by Lemma 1 in [7, if we let $\varepsilon \rightarrow 0$, 
it is easy to obtain, by diagonalization, a subsequence of $\left(v_{\varepsilon}, u_{\varepsilon}\right)_{\varepsilon>0}$ given by Proposition 1 , still denoted by $\left(v_{\varepsilon}, u_{\varepsilon}\right)_{\varepsilon>0}$, such that

$$
\begin{aligned}
& v_{\varepsilon} \underset{\varepsilon \rightarrow 0}{\rightarrow} v \text { in } L^{\infty}\left(\mathbf{R}_{+} ; L^{2}\right) \text { weak } \star \text { and a.e. in } \mathbf{R} \times \mathbf{R}_{+}, \\
& \left(v_{\varepsilon x}, v_{\varepsilon t}\right) \underset{\varepsilon \rightarrow 0}{\rightarrow}\left(v_{x}, v_{t}\right) \text { in }\left(L^{2}\left(\mathbf{R}_{+} ; L^{2}\right)\right)^{2} \text { weak, } u_{\varepsilon} \underset{\varepsilon \rightarrow 0}{\rightarrow} u \text { in } L^{\infty}\left(\mathbf{R}_{+} ; L^{2}\right) \text { weak } \text {, } \\
& u_{\varepsilon x} \underset{\varepsilon \rightarrow 0}{\rightarrow} u_{x} \text { in } L^{2}\left(\mathbf{R}_{+} ; L^{2}\right) \text { weak } \\
& \int_{-\varepsilon}^{v_{\varepsilon}} \rho_{\varepsilon}(y) d y \underset{\varepsilon \rightarrow 0}{\rightarrow} \theta \in \widetilde{H}(v) \text { a.e., and in } L^{\infty}\left(\mathbf{R} \times \mathbf{R}_{+}\right) \text {weak } \star
\end{aligned}
$$

and

Theorem 1. We have $v \in L^{\infty}\left(\mathbf{R}_{+} ; H^{1}\right) \cap C\left(\left[0,+\infty\left[; L^{2}\right), v_{x}, v_{t} \in L^{2}\left(\mathbf{R}_{+} ; L^{2}\right), u \in\right.\right.$ $L^{\infty}\left(\mathbf{R}_{+} ; L^{2}\right), u_{x} \in L^{2}\left(\mathbf{R}_{+} ; L^{2}\right), \theta \in L^{\infty}\left(\mathbf{R} \times \mathbf{R}_{+}\right), \theta \in \widetilde{H}(v)$ a.e. and

$$
\left\{\begin{array}{l}
\frac{\partial v}{\partial t}-\frac{\partial u}{\partial x}=0 \quad \text { in } \mathbf{R} \times \mathbf{R}_{+} \\
v(x, 0)=v_{0}(x) \quad \text { a.e. in } \mathbf{R} \\
\int_{0}^{+\infty} \int_{\mathbf{R}} u \frac{\partial \psi}{\partial t} d x d t-\int_{0}^{+\infty} \int_{\mathbf{R}}(\sigma(v)+\theta) \frac{\partial \psi}{\partial x} d x d t+\int_{\mathbf{R}} u_{0}(x) \psi(x, 0) d x \\
\quad=\mu \int_{0}^{+\infty} \int_{\mathbf{R}} \frac{\partial u}{\partial x} \frac{\partial \psi}{\partial x} d x d t, \quad \forall \psi \in C_{c}^{1}(\mathbf{R} \times[0,+\infty[) .
\end{array}\right.
$$

Hence, we say that $(v, u)$ is a weak solution of the Cauchy problem for the system

$$
\left\{\begin{array}{l}
v_{t}-u_{x}=0 \\
u_{t}-(\sigma(v)+H(v))_{x}=\mu u_{x x}
\end{array}\right.
$$

with initial data $\left(u_{0}, v_{0}\right) \in\left(H^{2}(\mathbf{R})\right)^{2}, v_{0} \in L^{1}(\mathbf{R})$.

Now, in the spirit of [10] (cf. [4] and [13] for related results), we look for the existence of shock profiles for system (1.10), that is, special solutions of the form

$$
(v, u)(x, t)=(V, U)\left(\frac{x-s t}{\mu}\right), \quad(V, U) \in\left(C(\mathbf{R}) \cap L^{\infty}(\mathbf{R})\right)^{2}
$$

for given $s \in \mathbf{R}$ such that, for certain pairs $\left(v_{-}, u_{-}\right),\left(v_{+}, u_{+}\right) \in \mathbf{R}^{2}$, we have

$$
\lim _{\xi \rightarrow-\infty}(V(\xi, U(\xi)))=\left(v_{-}, u_{-}\right), \quad \lim _{\xi \rightarrow+\infty}(V(\xi, U(\xi)))=\left(v_{+}, u_{+}\right) .
$$

We will choose $s, v_{-}, v_{+}$such that

$$
v_{+}<0<v_{-}, \quad-\sqrt{\frac{\sigma\left(v_{+}\right)}{v_{+}}}<s<-\sqrt{\sigma^{\prime}\left(v_{-}\right)}
$$

(notice that $\frac{\sigma\left(v_{+}\right)}{v_{+}} \leq \sigma^{\prime}\left(v_{+}\right)$, so (1.13) implies the Lax shock conditions, cf. [11]), and $u_{-}, u_{+}$such that (Rankine-Hugoniot conditions)

$$
\left\{\begin{array}{l}
-s\left(v_{+}-v_{-}\right)=u_{+}-u_{-} \\
-s\left(u_{+}-u_{-}\right)=\sigma\left(v_{+}\right)-\sigma\left(v_{-}\right)-1
\end{array}\right.
$$

(notice that $H\left(v_{+}\right)=0, H\left(v_{-}\right)=1$ ). Similarly, it is possible to consider the case $v_{-}<0<v_{+}$. 
Definition 1. We say that $(v, u) \in\left(L^{\infty}\left(\mathbf{R} \times \mathbf{R}_{+}\right)\right)^{2}$ is a weak solution of system (1.10) if there exists $\theta \in L^{\infty}\left(\mathbf{R} \times \mathbf{R}_{+}\right)$such that $\theta \in \widetilde{H}(v)$ a.e. and

$$
\left\{\begin{array}{l}
v_{t}-u_{x}=0, \\
u_{t}-(\sigma(v)+\theta)_{x}=\mu u_{x x},
\end{array} \text { in } \quad \mathcal{D}^{\prime}\left(\mathbf{R} \times \mathbf{R}_{+}\right) .\right.
$$

For special solutions of the form (1.11), (1.15) can be written as follows, with $\Theta \in L^{\infty}(\mathbf{R})$, $\Theta \in \widetilde{H}(v)$ a.e.,

$$
\left\{\begin{array}{l}
s V^{\prime}+U^{\prime}=0, \\
-s U^{\prime}-(\sigma(V)+\Theta)^{\prime}=U^{\prime \prime},
\end{array} \quad \text { in } \quad \mathcal{D}^{\prime}(\mathbf{R})\right.
$$

(where $V^{\prime}=\frac{d}{d \xi} V$ ).

We will prove the following theorem:

Theorem 2. Under the hypotheses (1.13), (1.14), the system (1.10) has a special weak solution $(v, u)$ of the form (1.11) verifying (1.12) (shock profile).

Now, for each $\mu>0$, let $\left(v_{\mu}, u_{\mu}\right)(x, t)=(V, U)\left(\frac{x-s t}{\mu}\right)$ be a shock profile in the framework of Theorem 2 and let $\mu \rightarrow 0^{+}$. For $x \neq s t,\left(v_{\mu}, u_{\mu}\right) \underset{\mu \rightarrow 0}{\longrightarrow}(v, u)$ defined by

$$
v(x, t)=\left\{\begin{array}{l}
v_{+} \text {for } x>s t, \\
v_{-} \text {for } x<s t,
\end{array} \quad u(x, t)=\left\{\begin{array}{l}
u_{+} \text {for } x>s t, \\
u_{-} \text {for } x<s t,
\end{array}\right.\right.
$$

and if $\theta_{\mu} \in \widetilde{H}\left(v_{\mu}\right)$ is as in Definition 1 , we derive $\theta_{\mu} \underset{\mu \rightarrow 0}{\longrightarrow} \theta$ (for $x \neq s t$ ) with

$$
\theta(x, t)=0 \text { for } x>s t, \quad \theta(x, t)=1 \text { for } x<s t,
$$

that is, $\theta(x, t)=1-H(x-s t), \theta \in \widetilde{H}(v)$ a.e.. Furthermore, it is easy to deduce (from (1.15) applied to $\left(v_{\mu}, u_{\mu}\right)$ and letting $\left.\mu \rightarrow 0\right)$

$$
\begin{aligned}
& \int_{0}^{+\infty} \int_{\mathbf{R}} v \varphi_{t} d x d t-\int_{0}^{+\infty} \int_{\mathbf{R}} u \varphi_{x} d x d t+\int_{\mathbf{R}} v(x, 0) \varphi(x, 0) d x \\
& +\int_{0}^{+\infty} \int_{\mathbf{R}} u \psi_{t} d x d t-\int_{0}^{+\infty} \int_{\mathbf{R}}(\sigma(v)+\theta) \psi_{x} d x d t+\int_{\mathbf{R}} u(x, 0) \psi(x, 0) d x=0
\end{aligned}
$$

for all $\varphi, \psi \in C_{0}^{1}(\mathbf{R} \times[0,+\infty[)$.

Hence, under the hypothesis of Theorem 2, we have solved the corresponding Riemann problem for the discontinuous $p$-system

$$
\left\{\begin{array}{l}
v_{t}-u_{x}=0 \\
u_{t}-(\sigma(v)+H(v))_{x}=0
\end{array}\right.
$$

in the sense introduced in [5]:

Theorem 3. Assume (1.13), (1.14). Then there exists a shock solution $(v, u)$, defined by (1.17), of the Riemann problem for (1.20). 


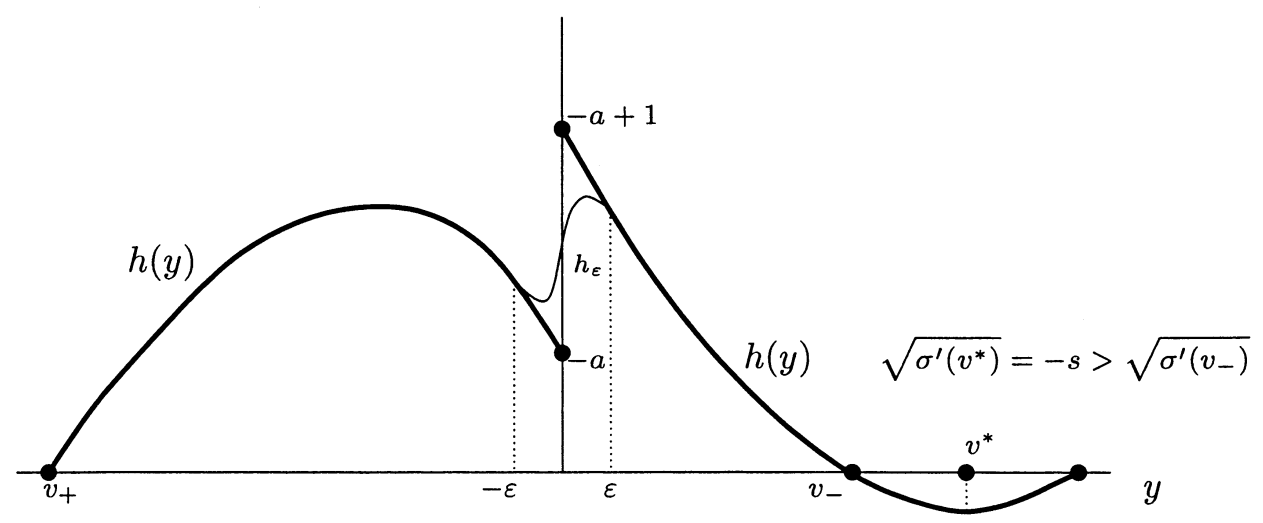

Fig. 1.

2. Proof of Theorem 2. For fixed $\mu>0, \varepsilon>0$, we look for a solution (we drop the $\varepsilon$ for simplicity) $(v, u)$ of the approximate system (1.5) in the form (1.11), $(V, U) \in$ $C^{1}(\mathbf{R}) \times C^{2}(\mathbf{R})$ verifying (1.12) under the hypothesis (1.13) and (1.14) by choosing $\varepsilon$ small enough such that $\rho_{\varepsilon}\left(v_{+}\right)=\rho_{\varepsilon}\left(v_{-}\right)=0$. System (1.5) reduces to (by setting $\xi=x-s t$ )

$$
\begin{gathered}
\left\{\begin{array}{l}
s V^{\prime}+U^{\prime}=0, \\
-s U^{\prime}-\left(\sigma^{\prime}(V)+\rho_{\varepsilon}(V)\right) V^{\prime}=U^{\prime \prime},
\end{array}\right. \\
s<0, \quad s^{2}=\frac{\sigma\left(v_{+}\right)-\sigma\left(v_{-}\right)-1}{v_{+}-v_{-}} .
\end{gathered}
$$

As in [10, Proposition 3.1, we derive, with $\sigma_{\varepsilon}$ defined by (1.4),

$$
\left\{\begin{array}{l}
s V-U=a_{1}=-s v_{ \pm}-u_{ \pm}, \\
-s U-\left(\sigma_{\varepsilon}(V)\right)=U^{\prime}+a_{2}, \quad a_{2}=-s u_{ \pm}-\sigma\left(v_{ \pm}\right)-H\left(v_{ \pm}\right),
\end{array}\right.
$$

and so

$$
s V^{\prime}=-s^{2} V+\sigma_{\varepsilon}(V)-a,
$$

with $a=s a_{1}-a_{2}=-s^{2} v_{ \pm}+\sigma\left(v_{ \pm}\right)+H\left(v_{ \pm}\right)$. Hence the function

$$
h_{\varepsilon}(y)=-s^{2} y+\sigma_{\varepsilon}(y)-a
$$

verifies $h_{\varepsilon}\left(v_{ \pm}\right)=0$. If we put $h(y)=-s^{2} y+\sigma(y)+H(y)-a$, we deduce

$$
h\left(v_{ \pm}\right)=0, \quad h\left(0^{-}\right)=-a, \quad h\left(0^{+}\right)=1-a \quad \text { and } \quad a<0,
$$

since $s^{2}<\frac{\sigma\left(v_{+}\right)}{v_{+}}$. It is easy to see that $\left.h(y)>0, h_{\varepsilon}(y)>0, y \in\right] v_{+}, v_{-}[$(cf. Fig. 1). Hence, following [10, Proof of Proposition 3.1, a solution $V_{\varepsilon}$ of the approximate problem (2.3) is given by

$$
s \int_{0}^{V_{\varepsilon}(\xi)} \frac{d y}{h_{\varepsilon}(y)}=\xi+c_{\varepsilon}
$$


and we can choose $c_{\varepsilon}=0$, that is, $V_{\varepsilon}(0)=0$. By $(2.2)$ we obtain the solution $\left(V_{\varepsilon}, U_{\varepsilon}\right)$. Now, for each $\varepsilon>0$ ( $\varepsilon$ small enough to have $\left.\rho_{\varepsilon}\left(v_{+}\right)=\rho_{\varepsilon}\left(v_{-}\right)=0\right)$ let

$$
\left(v_{\varepsilon}, u_{\varepsilon}\right)(x, t)=\left(V_{\varepsilon}, U_{\varepsilon}\right)\left(\frac{x-s t}{\mu}\right)
$$

be the corresponding shock profile. We know that $\left.V_{\varepsilon}(\xi) \in\right] v_{-}, v_{+}\left[, U_{\varepsilon}(\xi) \in\right] u_{-}, u_{+}[$. From (2.3), (2.2) and (2.1) we derive that

$$
\left\{\left(V_{\varepsilon}, U_{\varepsilon}\right)\right\}_{0<\varepsilon \leq \varepsilon_{0}} \text { is bounded in }\left(W^{1, \infty}(\mathbf{R})\right)^{2} .
$$

Hence, there is a subsequence, still denoted $\left\{\left(V_{\varepsilon}, U_{\varepsilon}\right)\right\}_{\varepsilon}$ and a pair $(V, U) \in(C(\mathbf{R}))^{2}$, such that $V(0)=0, V(\xi) \in\left[v_{-}, v_{+}\right], U(\xi) \in\left[u_{-}, u_{+}\right]$and

$$
V_{\varepsilon} \underset{\varepsilon \rightarrow 0}{\longrightarrow} V, \quad U_{\varepsilon} \underset{\varepsilon \rightarrow 0}{\longrightarrow} U
$$

uniformly in compact subsets of $\mathbf{R}$. In particular, by Lemma 1 in [7], we can assume that

$$
\int_{-\varepsilon}^{V_{\varepsilon}} \rho_{\varepsilon}(y) d y \underset{\varepsilon \rightarrow 0}{\rightarrow} \Theta \quad \text { in } \quad L^{\infty} \text { weak*, } \quad \Theta \in \widetilde{H}(V) \text { a.e. }
$$

We derive, by (2.1) written for $\left(V_{\varepsilon}, U_{\varepsilon}\right)$, by passing to the limit when $\varepsilon \rightarrow 0$,

$$
\left\{\begin{array}{l}
s V^{\prime}+U^{\prime}=0, \\
-s U^{\prime}-\frac{d}{d \xi}(\sigma(V)+\Theta)=U^{\prime \prime},
\end{array} \quad \text { in } \mathcal{D}^{\prime}(\mathbf{R}) .\right.
$$

It is easy to see that if

$$
v_{+}<V(\xi)<v_{-}, \quad \xi \in \mathbf{R},
$$

then

$$
s \int_{0}^{V_{\varepsilon}(\xi)} \frac{d y}{h_{\varepsilon}(y)} \underset{\varepsilon \rightarrow 0}{\longrightarrow} s \int_{0}^{V(\xi)} \frac{d y}{h(y)},
$$

and so

$$
s \int_{0}^{V(\xi)} \frac{d y}{h(y)}=\xi, \quad \xi \in \mathbf{R} .
$$

From the properties of $h$ (cf. [10] for related arguments) we derive

$$
V(\xi) \underset{\xi \rightarrow+\infty}{\longrightarrow} v_{+}, \quad V(\xi) \underset{\xi \rightarrow-\infty}{\longrightarrow} v_{-},
$$

and so (by passing also to the limit in $(2.2)$, written for $\left(V_{\varepsilon}, U_{\varepsilon}\right)$, when $\left.\varepsilon \rightarrow 0\right)$ we deduce

$$
U(\xi) \underset{\xi \rightarrow+\infty}{\longrightarrow} u_{+}, \quad U(\xi) \underset{\xi \rightarrow-\infty}{\longrightarrow} u_{-} .
$$

Finally, it is easy to conclude that (2.5) holds. This achieves the proof of Theorem 2.

Acknowledgements. This work was partially supported by FCT and FEDER through POCTI. The authors are indebted to Manuel Marques for interesting suggestions about mechanical models. 


\section{REFERENCES}

[1] J.F. Bell, The Experimental Foundations of Solid Mechanics, Encyclopedia of Physics, Vol VI a/1 (ed. by S. Flügge), Springer, 1973; Reprint of 1973 original, Springer-Verlag, Berlin, 1984. MR0763158 (86b:73001a)

[2] F. Caetano, On the existence of weak solutions to the Cauchy problem for a class of quasilinear hyperbolic equations with a source term, Rev. Mat. Complutense, 17 (2004), 147-167. MR2063946 (2005c:35198)

[3] J. Carrillo, Conservation laws with discontinuous flux functions and boundary condition, J. Evol. Equ. 3 (2003), 283-301). MR.1980978 (2004c:35266)

[4] C.M. Dafermos, Hyperbolic Conservation Laws in Continuum Physics, Springer, 2000. MR1763936 (2001m:35212)

[5] J.P. Dias and M. Figueira, On the Riemann problem for some discontinuous systems of conservation laws describing phase transitions, Comm. Pure Appl. Anal., 3 (2004), 53-58. MR2033459 (2004m:35179)

[6] J.P. Dias and M. Figueira, On the approximation of solutions of the Riemann problem for a discontinuous conservation law, Bull. Braz. Math. Soc., to appear.

[7] J.P. Dias, M. Figueira and J.F. Rodrigues, Solutions to a scalar discontinuous conservation law in a limit case of phase transitions, J. Math. Fluid. Mech., to appear.

[8] J. Greenberg, R. MacCamy and V. Mizel, On the existence, uniqueness, and stability of solutions of the equation $\sigma^{\prime}\left(u_{x}\right) u_{x x}+\lambda u_{x t x}=\rho_{0} u_{t t}$, J. Math. Mech., 17 (1968), 707-728. MR0225026 $(37: 623)$

[9] T. Gimse, Conservation laws with discontinuous flux functions, Siam J. Math. Anal., 24 (1993), 279-289. MR.1205526 (93j:35111)

[10] S. Kawashima and A. Matsumura, Stability of Shock Profiles in Viscoelasticity with Non-Convex Constitutive Relations, Comm. Pure Appl. Math., 47 (1994), 1547-1569. MR1303220 (95h:35136)

[11] P.G. LeFloch, Hyperbolic systems of conservation laws. The theory of classical and nonclassical shock waves, Lectures in Math. ETH Zürich, Birkhäuser, 2002. MR.1927887 (2003j:35209)

[12] P.D. Panagiotopoulos, Inequality Problems in Mechanics and Applications. Convex and Nonconvex Energy Functions, Birkhäuser, 1985. MR0896909 (88h:49003)

[13] D. Serre, Systèmes de Lois de Conservation, Diderot, 1996. MR1459988 (99b:35139)

[14] D. Serre and J. Shearer, Convergence with Physical Viscosity for Nonlinear Elasticity, preprint, Lyon, 1993, unpublished. 